\title{
Predicting Malignancy Risk in Gastrointestinal Subepithelial Tumors with Contrast-Enhanced Harmonic Endoscopic Ultrasonography Using Perfusion Analysis Software
}

\author{
Hyun Seok Lee, Chang Min Cho, Yong Hwan Kwon, and Su Youn Nam \\ Department of Internal Medicine, Kyungpook National University Hospital, School of Medicine, Kyungpook National University, Daegu, Korea
}

Background/Aims: Contrast-enhanced harmonic endoscopic ultrasonography (CEH-EUS) is a promising imaging modality that can differentiate subepithelial tumors (SETs) by detecting the degree of enhancement. However, whether $\mathrm{CEH}-$ EUS alone can predict the malignancy risk of gastrointestinal stromal tumors (GISTs) remains unclear. This study aimed to evaluate the feasibility of CEH-EUS by using perfusion analysis software for distinguishing among SETs and predicting the malignancy risk of GISTs. Methods: We retrospectively included patients with SETs who underwent preoperative CEH-EUS. In this study, 44 patients with histologically proven GISTs and benign SETs were enrolled. Perfusion analysis was performed using perfusion quantification software. Peak enhancement $(\mathrm{PE})$, wash-in rate $(\mathrm{WiR})$, wash-in perfusion index (WiPI), and wash-in and wash-out areas under the time-intensity curve (WiWoAUC) were calculated and compared between the GISTs and benign SETs. Results: When we allocated the enrolled patients into the leiomyoma group and low- and high-grade malignancy GIST groups, significant statistical differences in PE $(p<0.001)$, WiR $(p=0.009)$, WiPI $(p<0.001)$, and WiWoAUC $(p<0.001)$ were identified in the high-grade malignancy group compared with the leiomyoma group. Conclusions: CEH-EUS with perfusion analysis using perfusion analysis software could be a quantitative and independent method for predicting malignancy risk in gastrointestinal SETs. (Gut Liver 2019;13:161-168)

Key Words: Endosonography; Gastrointestinal stromal tumors; Contrast agent; Perfusion

\section{INTRODUCTION}

Gastrointestinal (GI) subepithelial tumors (SETs) are detected incidentally by endoscopy. GI SETs are of various types, with varied prognoses ranging from benign to aggressive and a malignant potential. Therefore, proper diagnostic and therapeutic approaches are required. ${ }^{1}$ When a GI SET is suspected, endoscopic ultrasonography (EUS) is the most accurate diagnostic method to determine the nature of the lesion, such as the originating layer, size, border, echogenic homogeneity, and the presence of echogenic and anechoic foci. ${ }^{1,2}$ However, most hypoechoic SETs are difficult to differentiate using EUS images alone. Gastrointestinal stromal tumors (GISTs) are the most commonly identified mesenchymal tumors in the stomach, and $10 \%$ to $30 \%$ of GISTs have a malignant potential. ${ }^{3,4}$ Although several features on EUS are specific for the prediction of the malignant potential of GISTs, the diagnostic accuracy of EUS findings varied widely, even with tissue sampling by EUSguided fine-needle aspiration, EUS guided Tru-Cut biopsy, and recently, EUS-guided fine-needle biopsy. ${ }^{1.5}$ Moreover, compared with computed tomography, EUS with contrast enhancement showed limited efficacy in characterizing lesions. ${ }^{6}$

Recent technical developments in contrast-enhanced harmonic EUS (CEH-EUS) with contrast agent have enabled the visualization of microvessels and parenchymal perfusion, which suggests the application of CEH-EUS to determine the malignancy risk of GISTs. ${ }^{4,6}$ Recent studies have shown that this technique helped to visualize intratumoral microvasculature or the pattern of contrast enhancement, which are important factors in determining the malignancy risk of GISTs. However, whether CEHEUS can predict the malignancy risk of GISTs remains unclear, which could be attributed to the difference in the interpretation

Correspondence to: Chang Min Cho (https://orcid.org/0000-0002-9903-1282)

Department of Internal Medicine, Kyungpook National University Hospital, School of Medicine, Kyungpook National University, 807 Hoguk-ro, Buk-gu, Daegu 41404, Korea

Tel: +82-53-200-2608, Fax: +82-53-200-2027, E-mail: cmcho@knu.ac.kr

Received on April 25, 2018. Revised on August 9, 2018. Accepted on August 10, 2018. Published online January 3, 2019

pISSN 1976-2283 eISSN 2005-1212 https://doi.org/10.5009/gnl18185

(a) This is an Open Access article distributed under the terms of the Creative Commons Attribution Non-Commercial License (http://creativecommons.org/licenses/by-nc/4.0) which permits unrestricted non-commercial use, distribution, and reproduction in any medium, provided the original work is properly cited. 
of CEH-EUS images according to the experience of the endoscopist and the equipment used. $4,5,7$

To overcome this limitation in the analysis of CEH-EUS measurements, the new perfusion analysis software VueBox (Bracco Suisse SA, Geneva, Switzerland) was introduced. ${ }^{8}$ Quantification of perfusion with CEH-EUS is feasible by analyzing the time sequence of contrast images and by calculating different perfusion parameters. ${ }^{9}$ A recent study has published the data of differential diagnosis for focal pancreatic lesions by using CEH-EUS quantification analyses. ${ }^{10}$ However, to our knowledge, no study published to date has evaluated the clinical usefulness of this method in patients with GI SETs. Therefore, this study aimed to evaluate the feasibility of CEH-EUS by using perfusion analysis software to distinguish SETs and to predict the malignancy risk of GISTs.

\section{MATERIALS AND METHODS}

\section{Patients}

We conducted a retrospective analysis and included patients with suspected SETs who were referred to Kyungpook National University Hospital for EUS examination from April 2012 to August 2017. All the patients were examined with B-mode EUS and CEH-EUS to analyze the characteristics of the respective SETs. All of them had provided informed consent before undergoing the procedures. The Institutional Review Board of our hospital approved this study (KNUMC_14-1024).

\section{Endosonographic evaluation}

Standard B-mode EUS was performed for all the patients with suspected subepithelial lesions. EUS was performed using a radial or linear echoendoscope (GF-UE260, GF-UCT260; Olympus Medical Systems, Tokyo, Japan) and a ProSound Alpha 10 processor (Aloka Co., Ltd., Tokyo, Japan).
A

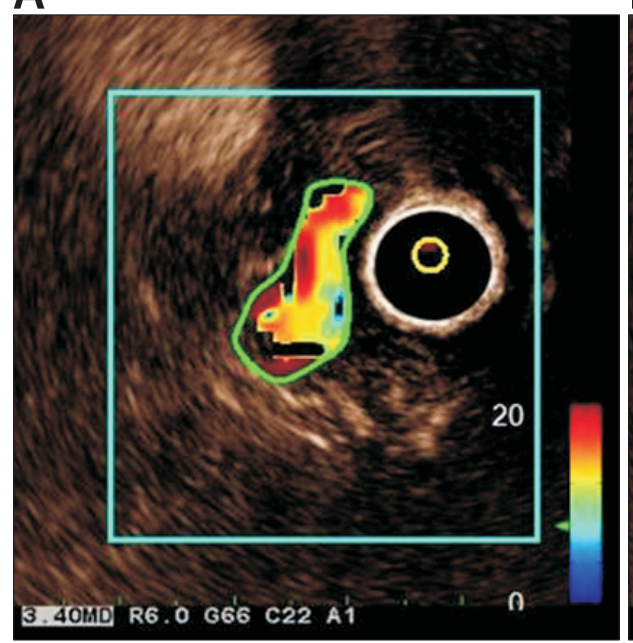

B

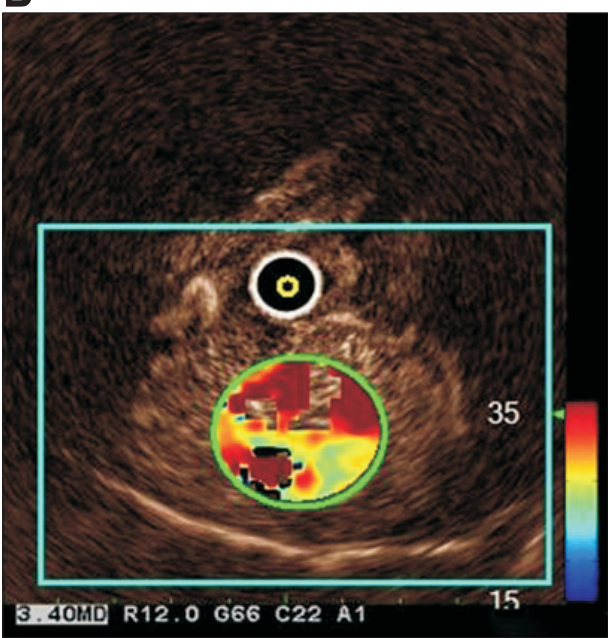

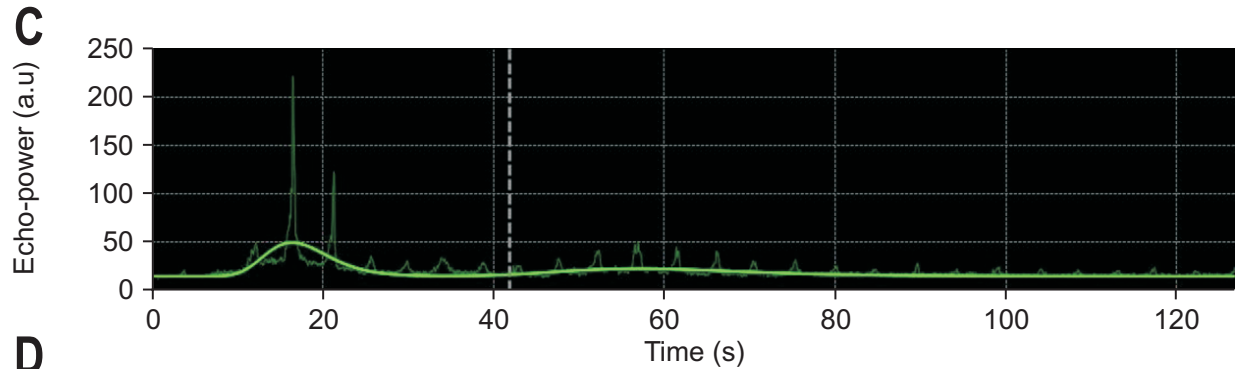

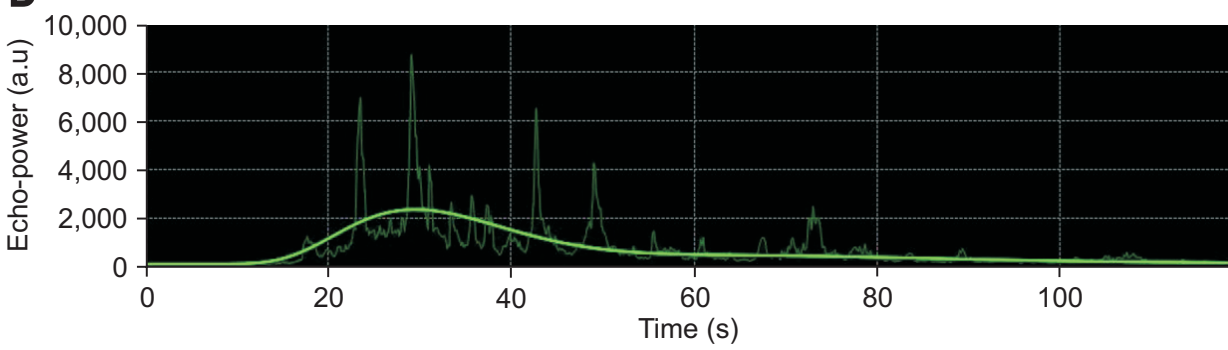

Fig. 1. Results screen of the quantitative parameters of CEH-EUS for leiomyoma and high-risk GIST of the stomach. The small reference region of interest (ROI) defines the baseline for quantitative analysis in the center of the CEH-EUS image, and the large ROI marks the hotspot in the enhanced tumor for (A) leiomyoma and (B) high-risk GIST. Time-intensity curves of the large ROI for the quantitative parameter "peak enhancement" for (C) leiomyoma and (D) high-risk GIST.

CEH-EUS, contrast-enhanced harmonic endoscopic ultrasonography; GIST, gastrointestinal stromal tumor; a.u., arbitrary units. 
For CEH-EUS, the extended pure harmonic detection mode was used, which combines the filtered fundamental and second harmonic component frequencies with a mechanical index of 0.17. ${ }^{4,5,11}$ When a subepithelial lesion was detected using fundamental B-mode EUS, the setting was changed to the extended pure harmonic detection mode. All the patients with solid subepithelial lesions received $2.4 \mathrm{~mL}$ of SonoVue (Bracco, Milan, Italy) as an ultrasonography contrast agent into the antecubital vein through a catheter, followed by $10 \mathrm{~mL}$ of saline flush. The CEH-EUS images were recorded for up to 120 seconds after the contrast injection. All B-mode EUS and CEH-EUS image clips were stored in the Digital Imaging and Communications in Medicine format, in the hard disk of the scanner, and were reviewed by a single experienced endosonographer (C.M.C.). The image clips were edited and evaluated using VueBox version 5.1. The software enabled the standardization of the quantification process. After uploading the image clips, calibration of the software and motion compensation are required to achieve sufficient reproducibility independent of the ultrasonography equipment used. A region of interest (ROI) was manually defined in all clip sequences by the same investigator (C.M.C.) and did not change throughout the clip (Fig. 1). ROI was set in part of the vascularized area of the lesions to analyze perfusion kinetics. The time-intensity curves generated by VueBox were automatically analyzed to determine the CEH-EUS parameters. The parameters for perfusion quantification include peak enhancement (PE) in arbitrary units (a.u), rise time (RT) in seconds, mean transit time (mTTI) in seconds, time to peak (TTP) in seconds, wash-in rate (WiR) in a.u, wash-in perfusion index (WiPI) in a.u, and area under time-intensity curve (AUC) in a.u. ${ }^{9,12-14}$

\section{Histopathological evaluation}

Among patients with SETs who underwent standard B-mode
EUS and CEH-EUS, those with histologically proven GISTs confirmed by surgical resection and those with histologically proven benign neoplasms confirmed by surgical resection or EUS-guided tissue sampling were enrolled. Each pathology report was evaluated to confirm the diagnosis and determine the National Institutes of Health classification in GISTs. ${ }^{15}$ GISTs were defined as SETs composed of spindle cells that stained positive for c-kit and CD34. The patients were categorized into three groups on the basis of their pathological reports as follows: patients with leiomyomas were categorized as a benign group, those with very low- and low-risk GISTs were defined as a low-grade malignancy group, and those with intermediateand high-risk GISTs were defined as a high-grade malignancy group. ${ }^{5}$

\section{Statistical analysis}

Categorical variables were expressed as numbers and percentages, and continuous variables were presented as medians and ranges, which were nonparametrically distributed. Statistical analyses of the differences in parameters for perfusion quantification among the three groups were conducted using the nonparametric Kruskal-Wallis test and the Mann-Whitney U test for comparisons between pairs of groups. Bonferroni correction was applied in the post hoc analysis of the intergroup comparisons. A p-value of $<0.05$ was considered statistically significant. Statistical calculations were performed using SPSS version 18.0 for the Windows software (SPSS Inc., Chicago, IL, USA).

\section{RESULTS}

\section{Baseline characteristics}

This study examined 44 patients, and their baseline characteristics are shown in Table 1. Patients with GISTs underwent sur-

Table 1. Baseline Characteristics of the Enrolled Patients

\begin{tabular}{|c|c|c|c|c|c|}
\hline \multirow[b]{2}{*}{ Characteristics } & \multirow{2}{*}{$\begin{array}{l}\text { Benign SETs* } \\
\quad(n=7)\end{array}$} & \multirow{2}{*}{$\begin{array}{l}\text { Leiomyoma } \\
\qquad(\mathrm{n}=5)\end{array}$} & \multicolumn{3}{|c|}{ GIST } \\
\hline & & & $\begin{array}{l}\text { Low-grade malignancy } \\
\qquad(\mathrm{n}=20)\end{array}$ & $\begin{array}{l}\text { High-grade malignancy } \\
\qquad(\mathrm{n}=12)\end{array}$ & p-value \\
\hline Male sex & 3 (42.9) & $2(40.0)$ & $8(40.0)$ & $6(50.0)$ & 0.848 \\
\hline Age, yr & $51(37-72)$ & $54(22-69)$ & $63(36-84)$ & $59(41-70)$ & 0.105 \\
\hline Location & & & & & 0.083 \\
\hline Esophagus & 0 & $2(40.0)$ & 0 & 0 & \\
\hline Stomach & 6 (85.7) & $3(60.0)$ & $17(85.0)$ & $9(75.0)$ & \\
\hline Duodenum & $1(14.3)$ & 0 & $1(5.0)$ & 2 (16.7) & \\
\hline Rectum & 0 & 0 & $2(10.0)$ & 1 (8.3) & \\
\hline Size, mm & $25(16-33)$ & $29(19-34)$ & $27(16-50)$ & $34(15-65)$ & 0.824 \\
\hline
\end{tabular}

Data are presented as number (\%) or median (range).

SETs, subepithelial tumors; GIST, gastrointestinal stromal tumor.

*Benign SETs included schwannoma $(n=3)$, glomus tumor $(n=2)$, ectopic pancreas $(n=1)$, and paraganglioma $(n=1)$. Low-grade malignancies included very low-risk GISTs $(\mathrm{n}=2)$ and low-risk GISTs $(\mathrm{n}=18)$. High-grade malignancies included intermediate risk GISTs $(\mathrm{n}=8)$ and high-risk GISTs $(\mathrm{n}=4) ;{ }^{\dagger} \mathrm{p}$-value was compared between leiomyoma, low- and high-grade malignancy GISTs. 
gery, and those with other tumors underwent surgery or EUSguided tissue sampling followed by histopathological analysis. The most common site of SETs was the stomach (79.5\%, 35/44). On the basis of their histological reports, 12 patients had benign tumors. In total, 32 patients (20 in the low-grade malignancy group and 12 in the high-grade malignancy group) were diagnosed as having GISTs.

In the CEH-EUS findings, a significant difference was found in the nonenhancing spots. Among the CEH-EUS features, nonenhancing spots were most common in the high-grade malignancy group (66.7\%), followed by the low-grade (55.0\%) and leiomyoma groups $(0 \% ; \mathrm{p}=0.028)$ (Table 2$)$.

\section{CEH-EUS data based on parameters for perfusion quanti- fication}

When comparing parameters for perfusion quantification between all benign tumors and GISTs, we found significantly higher TTP values for the GISTs than for the benign tumors (18.0 [14.9 to 690.0] vs 14.9 [10.2 to 17.1], $\mathrm{p}=0.029$ ) (Table 3).

In the comparison of the parameters for perfusion quantification between leiomyomas and GISTs, PE values were significantly higher for the GISTs than for the leiomyomas (860.2 [221.4 to $3,497.7$ ] vs 114.8 [50.7 to 423.4], $p=0.011$ ). The WiR and WiPI values for the GISTs were significantly higher than those for the leiomyomas (WiR: 17.0 [9.8 to 118.4 ] vs 148.5 [45.4 to 690.0]; $p=0.024$ and WiPI: 73.3 [31.3 to 266.5] vs 540.5

Table 2. Standard B-Mode and Contrast-Enhanced Harmonic Endoscopic Ultrasonographic Features

\begin{tabular}{|c|c|c|c|c|}
\hline Features & Leiomyoma $(n=5)$ & $\begin{array}{l}\text { Low-grade malignancy GIST } \\
\qquad(\mathrm{n}=20)\end{array}$ & $\begin{array}{l}\text { High-grade malignancy GIST } \\
\qquad(\mathrm{n}=12)\end{array}$ & p-value \\
\hline \multicolumn{5}{|c|}{ Standard B-mode EUS findings } \\
\hline Size, $\mathrm{mm}$ & $29(19-34)$ & $27(16-50)$ & $34(15-65)$ & 0.824 \\
\hline Heterogeneity & $3(60.0)$ & $11(55.0)$ & $9(75.0)$ & 0.397 \\
\hline Irregular margin & $1(20.0)$ & $2(10.0)$ & $3(25.0)$ & 0.559 \\
\hline \multicolumn{5}{|l|}{ CEH-EUS findings } \\
\hline Irregular vessels & 0 & $11(55.0)$ & $8(66.7)$ & 0.028 \\
\hline Heterogeneous perfusion & $2(40.0)$ & $12(60.0)$ & $5(6.2)$ & 0.767 \\
\hline Hyperechoic foci & $1(20.0)$ & $10(50.0)$ & $8(66.7)$ & 0.090 \\
\hline Nonenhancing spots & 0 & $11(55.0)$ & 8 (66.7) & $0.028^{*}$ \\
\hline
\end{tabular}

Data are presented as median (range) or number (\%).

GIST, gastrointestinal stromal tumor; EUS, endoscopic ultrasonography; CEH-EUS, contrast-enhanced harmonic EUS.

*Linear-by-linear association.

Table 3. Comparison of Parameters for Perfusion Quantification between Benign and Gastrointestinal Stromal Tumors

\begin{tabular}{lccc}
\hline Parameters & Benign SETs $(\mathrm{n}=12)^{*}$ & GISTs $(\mathrm{n}=32)$ & $\mathrm{p}$-value \\
\hline PE & $419.9(128.0-1,049.1)$ & $860.2(221.4-3497.7)$ & 0.511 \\
WiAUC & $1,730.5(610.7-6,241.1)$ & $4,239.1(949.3-14,802.5)$ & 0.299 \\
RT & $6.4(5.1-9.2)$ & $7.3(6.2-8.3)$ & 0.465 \\
mTTI & $30.3(19.3-73.5)$ & $33.1(21.8-58.6)$ & 0.860 \\
TTP & $14.9(10.2-17.1)$ & $18.0(14.9-690.0)$ & 0.029 \\
WiR & $102.2(19.8-191.1)$ & $148.5(45.4-690.0)$ & 0.972 \\
WiPI & $260.3(80.9-652.5)$ & $540.5(137.0-2,143.5)$ & 0.538 \\
WoAUC & $2,620.5(979.3-9,800.6)$ & $6,880.9(1,370.6-19,831.9)$ & 0.408 \\
WiWoAUC & $4,351.0(1,590.0-15,997.0)$ & $11,424.5(2,319.9-34,634.4)$ & 0.361 \\
FT & $9.4(8.6-15.9)$ & $10.2(8.5-13.8)$ & 0.867 \\
WoR & $50.7(9.0-100.9)$ & $77.3(20.4-445.6)$ & 0.549 \\
QOF & $64.0(34.9-84.1)$ & $67.2(46.5-78.9)$ & 0.924 \\
\hline
\end{tabular}

Data are presented as median (interquartile range).

SETs, subepithelial tumors; GISTs, gastrointestinal stromal tumors; PE, peak enhancement; WiAUC, wash-in area under the curve; RT, rise time; mTTI, mean transit time; TTP, time to peak; WiR, wash-in rate; WiPI, wash-in perfusion index; WoAUC, wash-out area under the curve; WiWoAUC, wash-in/wash-out area under the curve; FT, fall time; WoR, wash-out rate; QOF, quality of fit. *Including leiomyoma $(n=5)$, schwannoma $(n=3)$, glomus tumor $(n=2)$, ectopic pancreas $(n=1)$, and paraganglioma $(n=1)$. 

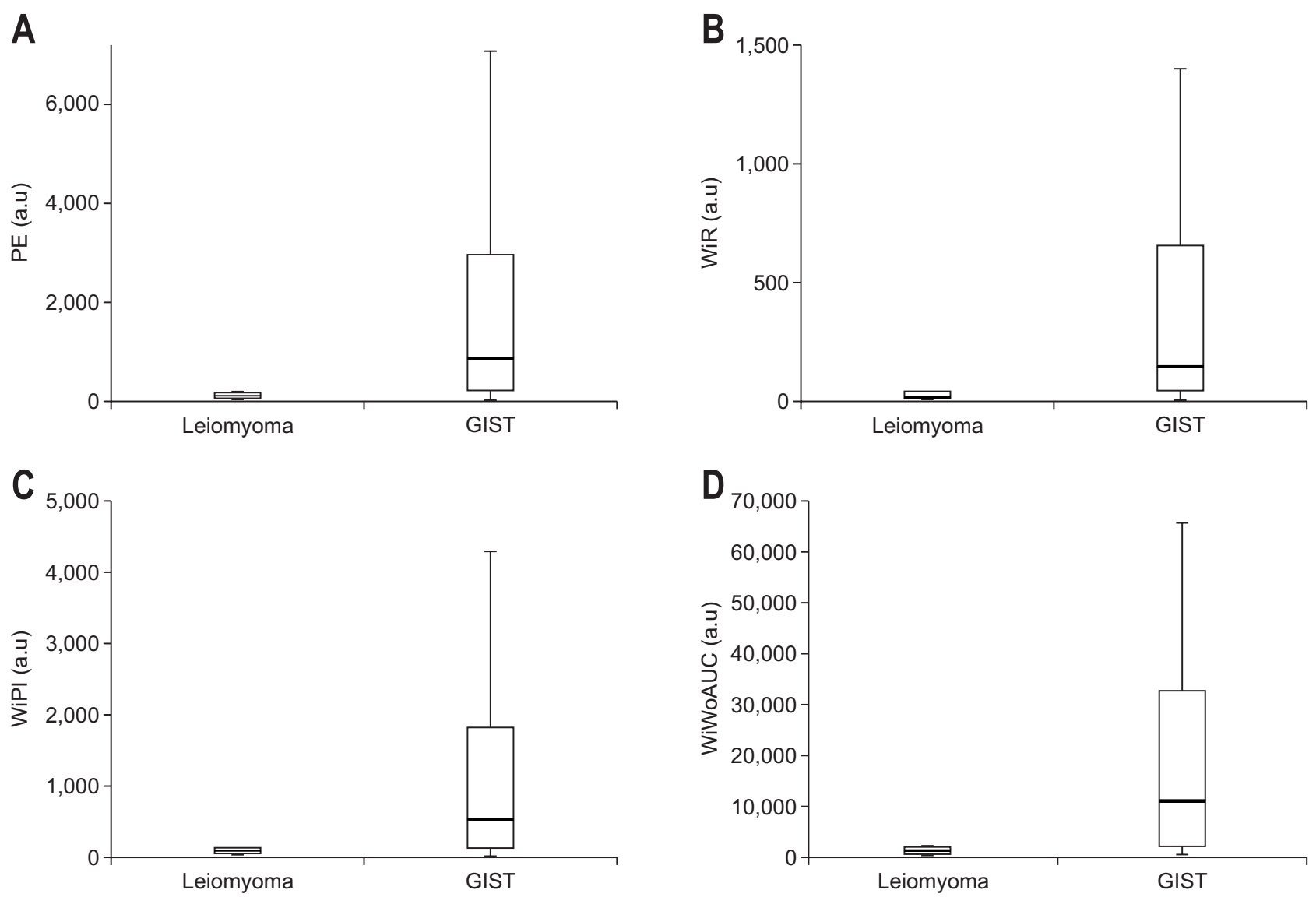

Fig. 2. Parameters quantified using CEH-EUS measurements for leiomyomas and GISTs with significant differences ( $<<0.05)$ : $(A) P E(p=0.010),(B)$ WiR ( $p=0.024)$, (C) WiPI ( $p=0.012)$, and (D) WiWoAUC $(p=0.009)$.

CEH-EUS, contrast-enhanced harmonic endoscopic ultrasonography; GIST, gastrointestinal stromal tumor; PE, peak enhancement; a.u., arbitrary units; WiR, wash-in rate; WiPI, wash-in perfusion index; WiWoAUC, wash-in and wash-out area under the curve.

[137.0 to 2,143.5], $p=0.012$ ) (Fig. 2). Moreover, the wash-in and wash-out AUC (WiWoAUC) values were significantly higher in the GISTs than in the leiomyomas $(1,424.8$ [519.1 to 3,917.1] vs $11,424.5$ [2,319.9 to $34,634.4], \mathrm{p}=0.006)$. No significant differences were found with regard to RT and mTTI.

In the comparison of the perfusion parameters of CEH-EUS in the leiomyoma, low-, and high-grade malignancy groups, the values of PE, WiR, WiPI, and WiWoAUC were significantly higher in the high-grade malignancy group than in the leiomyoma group ( $\mathrm{p}=0.001, \mathrm{p}=0.009, \mathrm{p}=0.001$, and $\mathrm{p}=0.001$, respectively) (Table 4, Fig. 3). However, no significant difference was found with regard to RT and mTTI.

We also analyzed the diagnostic performance (sensitivity, specificity, positive predictive value, and negative predictive value) of each parameter for perfusion quantification for the GISTs in comparison with the leiomyomas (Table 5). By using the cutoff values of the parameters, the sensitivities of PE, WiR, WiPI, and WiWoAUC ranged from $78.1 \%$ to $84.4 \%$. The specificity of these four parameters was 60\%.

\section{DISCUSSION}

To determine the malignancy risk of GISTs, surgical resection is generally required to detect the tumor size and mitotic count. However, some studies have shown that the absolute potential of malignancy in small GISTs appears to be very low and that only $1.9 \%$ of patients with very low risk showed disease progression during follow-up. ${ }^{15,16}$ Therefore, whether surgical resection should be performed to confirm the malignancy of low-risk GISTs has been unclear. ${ }^{5}$ This has prompted research for alternative approaches to preoperatively predict malignancy risk.

CEH-EUS is a new promising EUS technique for analysis of tumor microcirculation. This dynamic examination with a high temporal solution allows detailed description of the vascular architecture in the tumor tissue after intravenous administration of microbubbles as a contrast agent. ${ }^{9,17,18}$ Standardized measurements using CEH-EUS as a reproducible and valid method are required in clinical practice because analysis of tumor microcirculation and changes in contrast enhancements should not differ among EUS experts. Therefore, a standardized analysis of tumor perfusion might be helpful. ${ }^{9}$ 
Table 4. Comparison of Parameters for Perfusion Quantification in Gastrointestinal Subepithelial Tumors among the Leiomyoma, Low-Grade Malignancy, and High-Grade Malignancy Groups

\begin{tabular}{|c|c|c|c|c|c|c|c|}
\hline \multirow{2}{*}{ Parameters } & \multirow{2}{*}{$\begin{array}{l}\text { Leiomyoma } \\
\quad(n=5)\end{array}$} & \multirow{2}{*}{$\begin{array}{l}\text { Low-grade malignancy } \\
\qquad(\mathrm{n}=20)\end{array}$} & \multirow{2}{*}{$\begin{array}{l}\text { High-grade malignancy } \\
\qquad(\mathrm{n}=12)\end{array}$} & \multicolumn{4}{|c|}{ p-value } \\
\hline & & & & Overall & $\mathrm{A}^{*}$ & $\mathrm{~B}^{\dagger}$ & $\mathrm{C}^{\ddagger}$ \\
\hline PE & $114.8(50.7-423.4)$ & $608.8(169.3-3,497.7)$ & 1,202.9 (846.9-3,500.8) & 0.011 & 0.060 & 0.001 & 0.099 \\
\hline WiAUC & $575.7(214.0-1,401.6)$ & $2,619.9(620.6-15,225.0)$ & $5,634.7(3665.4-14,802.5)$ & 0.007 & 0.042 & 0.001 & 0.076 \\
\hline RT & $6.2(5.3-8.7)$ & $7.2(5.8-8.0)$ & $7.8(6.4-9.7)$ & 0.391 & 0.692 & 0.195 & 0.346 \\
\hline mTTI & $28.4(23.1-107.3)$ & $32.8(20.2-65.8)$ & $34.7(23.5-57.8)$ & 0.849 & 0.869 & 0.959 & 0.578 \\
\hline ТTP & $14.5(9.9-16.0)$ & $18.5(16.5-20.5)$ & $15.8(12.9-18.2)$ & 0.016 & 0.006 & 0.234 & 0.076 \\
\hline WiR & $17.0(9.8-118.5)$ & $104.7(24.1-690.0)$ & $206.0(107.1-726.2)$ & 0.029 & 0.083 & 0.009 & 0.146 \\
\hline WiPI & $73.3(31.3-266.5)$ & $377.5(105.1-2,143.5)$ & $743.7(534.2-2,140.0)$ & 0.013 & 0.071 & 0.001 & 0.107 \\
\hline WoAUC & $849.1(305.1-2,515.5)$ & $3,473.4(836.5-20,761.7)$ & $9,007.2(6871.2-19,831.9)$ & 0.009 & 0.112 & 0.001 & 0.053 \\
\hline WiWoAUC & $1,424.8(519.1-3,917.1)$ & 6,093.2 (1484.9-36,047.8) & $14,401.9(11333.6-34,634.4)$ & 0.006 & 0.060 & 0.001 & 0.053 \\
\hline FT & $9.2(8.7-16.1)$ & $9.9(8.3-11.3)$ & $12.9(9.3-16.1)$ & 0.280 & 0.668 & 0.442 & 0.125 \\
\hline WoR & $7.1(5.5-52.5)$ & $72.2(13.9-445.6)$ & $108.1(59.7-484.0)$ & 0.039 & 0.051 & 0.014 & 0.289 \\
\hline QOF & $58.3(35.5-77.0)$ & $64.5(36.4-78.9)$ & $70.7(52.0-79.5)$ & 0.770 & 0.767 & 0.506 & 0.659 \\
\hline
\end{tabular}

Data are presented as median (interquartile range).

PE, peak enhancement; WiAUC, wash-in area under the curve; RT, rise time; mTTI, mean transit time; TTP, time to peak; WiR, wash-in rate; WiPI, wash-in perfusion index; WoAUC, wash-out area under the curve; WiWoAUC, wash-in/wash-out area under the curve; FT, fall time; WoR, washout rate; QOF, quality of fit.

*Leiomyoma vs low-grade malignancy; ${ }^{\dagger}$ Leiomyoma vs high-grade malignancy; ${ }^{\ddagger}$ Low-grade malignancy vs high-grade malignancy. ABC $p$-value: p-values obtained from a Kruskal-Wallis test when comparing three groups and Mann-Whitney U test and Bonferroni's correction for multiple comparisons between two groups.

In this study, we analyzed patients in leiomyoma, low-grade, and high-grade malignancy GIST groups from among those with GI SETs by using perfusion analysis software for the quantification of EUS perfusion-related parameters.

While RT and mTTI showed no significant differences when GISTs were compared with leiomyomas, PE, WiR, WiPI, and WiWoAUC showed statistically significant higher values for GISTs in this study. Abnormal blood vessels are considered one of the major factors in distinguishing GISTs from leiomyomas and in the prediction of malignant risk. ${ }^{19}$ Although CEH-EUS is beneficial for the detection of blood flow in abnormal intratumoral blood vessels, ${ }^{19}$ it has a limitation in that the pattern analysis depends on the operator subjectively. However, CEHEUS with perfusion analysis using perfusion analysis software revealed various objective perfusion parameters that GISTs had more blood flow of abnormal angiogenesis within the tumors. Therefore, CEH-EUS with quantitative perfusion analysis revealed a different pattern with higher blood flow in GISTs than in leiomyomas.

Compared with that of leiomyoma, the high-risk malignancy of GIST showed significantly higher values for PE, WiR, WiPI, and WiWoAUC. However, no significant differences were observed between the leiomyoma and low-risk malignancy groups and between the low- and high-risk malignancy groups. This result might be attributed to the widely distributed data of the low-risk malignancy group, although the median values for each parameter were different among the three groups.
To our knowledge, this is the first study to describe the prediction of malignancy risk in GI SETs with CEH-EUS by using perfusion analysis software (VueBox), based on histological evaluation.

This study has some limitations. First, it was a retrospective study. We collected data from GI SETs after evaluating CEHEUS and reviewed the data using perfusion software. Patient selection bias might have existed because the data were selected on the basis of histologically proven SETs. Nonetheless, we tried to minimize selection bias by including all patients who had undergone surgery or EUS-guided tissue sampling after CEHEUS. Second, our study was limited by the small number of patients and did not show significant differences between the leiomyoma and low-risk malignancy groups and between the low- and high-risk malignancy groups. We believe that this can be overcome by recruiting a large number of patients with SETs in future studies. Third, the ROI was not placed at the normal thin GI tissue in this study, although ROIs were compared at both the lesion and normal parenchyma in previous studies on other organs such as the pancreas and analyzed using VueBox. Complementary software or further studies might be required to address this aspect. Despite these limitations, we suggest that the present study has value because the prediction of malignancy risk in GI SETs with CEH-EUS was evaluated for the first time by using perfusion analysis software.

In conclusion, our results indicate that parameters such as PE, WiR, WiPI, and AUC significantly differ between the high-grade 

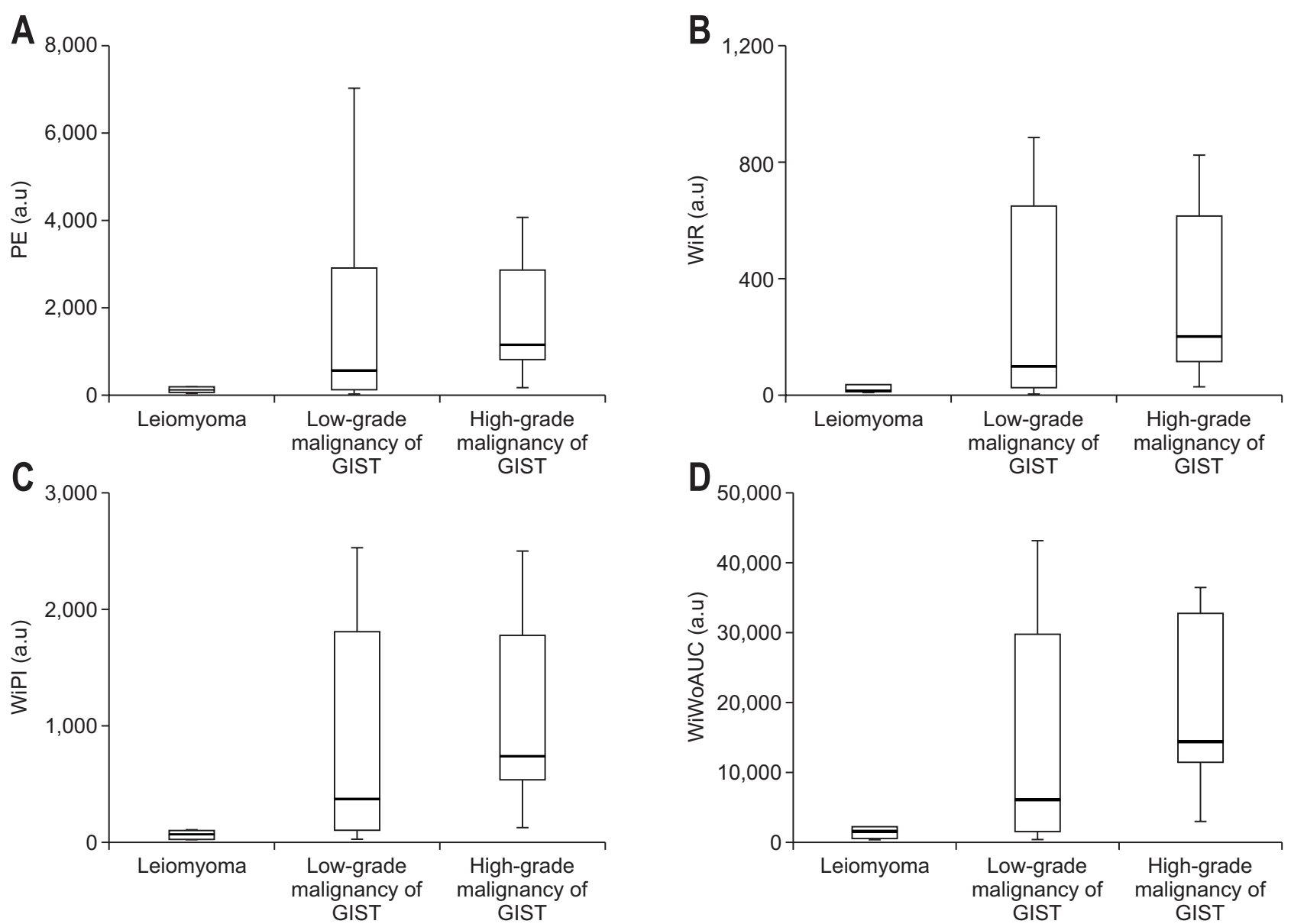

Fig. 3. Parameters quantified using CEH-EUS measurements concerning leiomyoma, low-grade malignancies of GISTs, and high-grade malignancies of GISTs. (A) PE, (B) WiR, (C) WiPI, and (D) WiWoAUC ( $p<0.001$ at PE, $p=0.009$ at WiR, $p<0.001$ at WiPI, and $p<0.001$ at WiWoAUC between the leiomyoma and high-grade malignancy GIST groups).

CEH-EUS, contrast-enhanced harmonic endoscopic ultrasonography; GIST, gastrointestinal stromal tumor; PE, peak enhancement; a.u., arbitrary units; WiR, wash-in rate; WiPI, wash-in perfusion index; WiWoAUC, wash-in/wash-out area under the curve.

Table 5. Sensitivity, Specificity, and Odds Ratio of the Parameters for Perfusion Quantification for GISTs in Compared with Leiomyomas

\begin{tabular}{|c|c|c|c|c|c|c|}
\hline Parameter & Cutoff value & Sensitivity & Specificity & $\begin{array}{l}\text { Positive predictive } \\
\text { value }\end{array}$ & $\begin{array}{l}\text { Negative predictive } \\
\text { value }\end{array}$ & Odds ratio \\
\hline PE & 166.7 & 84.4 & 60.0 & 93.1 & 37.5 & 8.1 \\
\hline WiR & 37.3 & 78.1 & 60.0 & 92.6 & 30.0 & 5.4 \\
\hline WiPI & 103.35 & 84.4 & 60.0 & 93.1 & 37.5 & 8.1 \\
\hline WiWoAUC & 2037.5 & 78.1 & 60.0 & 92.6 & 30.0 & 5.4 \\
\hline
\end{tabular}

GISTs, gastrointestinal stromal tumors; PE, peak enhancement; a.u., arbitrary units; WiR, wash-in rate; WiPI, wash-in perfusion index; WiWoAUC, wash-in/wash-out area under the curve.

malignancy and benign leiomyoma groups in a perfusion analysis quantified using CEH-EUS measurements. CEH-EUS with a perfusion analysis using perfusion analysis software could be a quantitative and independent method for predicting malignancy risk in GI SETs. We hope that this study will lead to prospective studies with larger numbers of patients to elucidate the exact role of CEH-EUS with a perfusion analysis in the assessment of GI SETs.

\section{CONFLICTS OF INTEREST}

No potential conflict of interest relevant to this article was reported.

\section{ACKNOWLEDGEMENTS}

This work was supported by a Biomedical Research Institute 
grant from Kyungpook National University Hospital (2014).

\section{REFERENCES}

1. Moon JS. Role of endoscopic ultrasonography in guiding treatment plans for upper gastrointestinal subepithelial tumors. Clin Endosc 2016;49:220-225.

2. Moon JS. Endoscopic ultrasound-guided fine needle aspiration in submucosal lesion. Clin Endosc 2012;45:117-123.

3. Miettinen M, Sarlomo-Rikala M, Lasota J. Gastrointestinal stromal tumors: recent advances in understanding of their biology. Hum Pathol 1999;30:1213-1220.

4. Sakamoto H, Kitano M, Matsui S, et al. Estimation of malignant potential of GI stromal tumors by contrast-enhanced harmonic EUS (with videos). Gastrointest Endosc 2011;73:227-237.

5. Park HY, Jeon SW, Lee HS, et al. Can contrast-enhanced harmonic endosonography predict malignancy risk in gastrointestinal subepithelial tumors? Endosc Ultrasound 2016;5:384-389.

6. Kitano M, Sakamoto H, Kudo M. Contrast-enhanced endoscopic ultrasound. Dig Endosc 2014;26 Suppl 1:79-85.

7. Yamashita Y, Kato J, Ueda K, et al. Contrast-enhanced endoscopic ultrasonography can predict a higher malignant potential of gastrointestinal stromal tumors by visualizing large newly formed vessels. J Clin Ultrasound 2015;43:89-97.

8. Tranquart F, Mercier L, Frinking P, Gaud E, Arditi M. Perfusion quantification in contrast-enhanced ultrasound (CEUS): ready for research projects and routine clinical use. Ultraschall Med 2012;33 Suppl 1:S31-S38.

9. Klotz LV, Ingrisch M, Eichhorn ME, et al. Monitoring parotid gland tumors with a new perfusion software for contrast-enhanced ultrasound. Clin Hemorheol Microcirc 2014;58:261-269.

10. Gheonea DI, Streba CT, Ciurea T, Săftoiu A. Quantitative low mechanical index contrast-enhanced endoscopic ultrasound for the differential diagnosis of chronic pseudotumoral pancreatitis and pancreatic cancer. BMC Gastroenterol 2013;13:2.

11. Săftoiu A, Dietrich CF, Vilmann P. Contrast-enhanced harmonic endoscopic ultrasound. Endoscopy 2012;44:612-617.

12. Zink F, Kratzer W, Schmidt S, et al. Comparison of two high-end ultrasound systems for contrast-enhanced ultrasound quantification of mural microvascularity in Crohn's disease. Ultraschall Med 2016;37:74-81.

13. Wilkens R, Peters DA, Nielsen AH, Hovgaard VP, Glerup H, Krogh K. Dynamic contrast-enhanced magnetic resonance enterography and dynamic contrast-enhanced ultrasonography in Crohn's disease: an observational comparison study. Ultrasound Int Open 2017;3:E13-E24.

14. Wildner D, Pfeifer L, Goertz RS, et al. Dynamic contrast-enhanced ultrasound (DCE-US) for the characterization of hepatocellular carcinoma and cholangiocellular carcinoma. Ultraschall Med 2014;35:522-527.

15. Fletcher CD, Berman JJ, Corless C, et al. Diagnosis of gastrointestinal stromal tumors: a consensus approach. Hum Pathol 2002;33:459-465.

16. Miettinen M, Lasota J. Gastrointestinal stromal tumors: pathology and prognosis at different sites. Semin Diagn Pathol 2006;23:7083.

17. Clevert DA, D’Anastasi M, Jung EM. Contrast-enhanced ultrasound and microcirculation: efficiency through dynamics: current developments. Clin Hemorheol Microcirc 2013;53:171-186.

18. Eichhorn ME, Klotz LV, Luedemann S, et al. Vascular targeting tumor therapy: non-invasive contrast enhanced ultrasound for quantitative assessment of tumor microcirculation. Cancer Biol Ther 2010;9:794-802.

19. Chhoda A, Jain D, Surabhi VR, Singhal S. Contrast enhanced harmonic endoscopic ultrasound: a novel approach for diagnosis and management of gastrointestinal stromal tumors. Clin Endosc 2018;51:215-221. 